\title{
Late breaking abstracts from the 6th international conference on prevention \& infection control (ICPIC 2021)
}

\author{
Geneva, Switzerland. 14-17 September 2021 \\ Published: 29 December 2021
}

\section{Slide session: Late breaking abstracts}

\section{5}

Burden of Covid-19 in Long-Term-Care Facilities: Preliminary Results of a Multicentre Cohort-Study from Eastern and Western Switzerland G. Scanferla 1,*, N. Graf'2, D. Héquet ${ }^{3}$, S. Kessler ${ }^{1}$, P. Kohler ${ }^{1}$, A. Nussbaumer ${ }^{1}$, M. Schlegel', D. Flury

${ }^{1}$ Department of Infectious Diseases and Hospital Epidemiology, ${ }^{2}$ Clinical Trials Unit (CTU), Canton Hospital St. Gallen, St. Gallen, ${ }^{3}$ Unité cantonale HPCi Vaud, Lausanne, Switzerland

Antimicrobial Resistance \& Infection Control 2021, 10(2): 045

Background: COVID-19 outbreaks with high mortality rates have been reported from long-term care facilities (LTCFs) worldwide despite infection control and prevention (ICP) measures. However, no systematic data exist on COVID-19 burden and its risk factors in Swiss-LTCFs.

Aims: to assess (i) COVID-19 incidence and -related mortality for the year 2020 and their influencing factors, (ii) vaccination rates of residents and health care workers (HCW).

Methods: LTCFs from cantons of St Gallen (SG) and Vaud (VD) (Eastern and Western Switzerland) were invited to participate in this cohort study. In May 2021, we collected COVID-19 cases and -related deaths 2020 and their potential risk factors at institutional level (e.g. size, number of single beds, staffing, ICP measures, aggregated resident characteristics) as well as vaccination rates of residents and HCW. Risk factor analysis and prospective surveillance of COVID-19 is ongoing. Results: We enrolled a total of 59 institutions ( 33 from SG, 26 from VD), with a median of 46 (IQR 33-69) occupied beds. In 2020, median COVID19 incidence was 38.6 per 100 occupied beds, with higher rates in VD than in SG ( $48 \%$ vs. $29.4 \%, p=0.028)$ (Fig. 1). Rates varied widely among LTCFs, with some institutions reporting no COVID-19 cases. On average (median), one-fifth (20\%) of COVID-19 cases died and COVID-19 related deaths accounted for $21.6 \%$ of total deaths. Regarding ICP measures, $54 / 59$ institutions (91.5\%) recommended using gowns in contact with COVID-19 patients, whilst use of other PPE (i.e. FFP2 masks, glows and googles) was more heterogeneous. Only a few LTCFs reported having ever used regular testing of asymptomatic residents $(6 / 59,10.2 \%)$ or HCW $(14 / 59,23.7 \%)$ as a prevention strategy. Nearly half of them $(27 / 59$, $45.8 \%)$ never banned visits or only in outbreak situations. Of 2786 residents, a median of $72.2 \%$ per facility received $\geq 1$ dose of COVID-19 vaccine. COVID-19 vaccine uptake among HCW was low (28.6\%) (Fig. 1).

\begin{abstract}
Figure / Image:
Figure 1 A COVID-19 incidence, total deaths and COVID-19 related mortality, 2020. Surviving COVID-19 cases (yellow bars), COVID-19 related deaths (blue bars) and total deaths (blank bars) per occupied beds and institutions are shown. Horizontal lines represent the median of total COVID-19 cases (red line), COVID-19 related deaths (blue line) and total deaths (black line). COVID-19 rates $>100 \%$ indicate high turnover rates of COVID-19 affected residents. B COVID-19 vaccination rates per institution among residents (black dots) and HCWs (red dots), as for May 2021. Horizontal lines show the median of vaccination rate of residents (black line) and HCWs (red line). Institutions are in the same order as in panel A
\end{abstract}

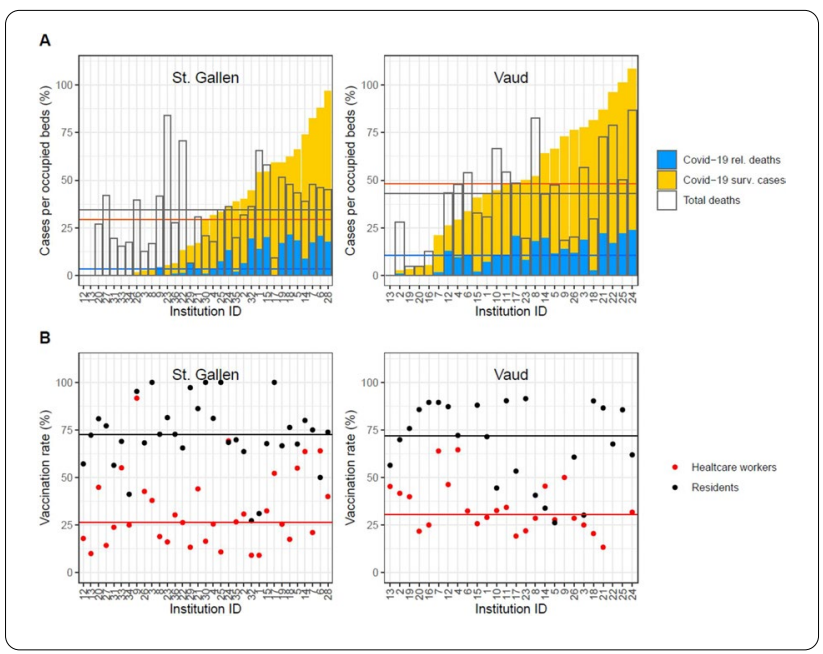

Conclusion: COVID-19 burden was highly heterogeneous in Swiss LTCFs. Further analyses will reveal factors, which could potentially explain these differences. Of note, we found a relatively high COVID-19 vaccine uptake among care home residents, whilst vaccine coverage of $\mathrm{HCW}$ is low.

Disclosure of interest

None declared.

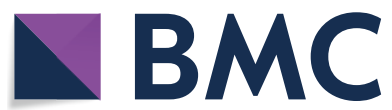

(c) The Author(s) 2021. Open Access This article is licensed under a Creative Commons Attribution 4.0 International License, which permits use, sharing, adaptation, distribution and reproduction in any medium or format, as long as you give appropriate credit to the original author(s) and the source, provide a link to the Creative Commons licence, and indicate if changes were made. The images or other third party material in this article are included in the article's Creative Commons licence, unless indicated otherwise in a credit line to the material. If material is not included in the article's Creative Commons licence and your intended use is not permitted by statutory regulation or exceeds the permitted use, you will need to obtain permission directly from the copyright holder. To view a copy of this licence, visit http://creativecommons.org/licenses/by/4.0/. The Creative Commons Public Domain Dedication waiver (http://creativecommons.org/publicdomain/zero/1.0/) applies to the data made available in this article, unless otherwise stated in a credit line to the data. 
046

Out of a Comfort Zone: Challenges of Infection Prevention in a 4000 Bedded Intensive Care Unit Built in an Exhibition Centre M. Kiernan 1,* on behalf of Infection Prevention and Control Team, London Nightingale Hospital

'Infection Prevention and Control, UNIVERSITY OF WEST LONDON, London, United Kingdom

Antimicrobial Resistance \& Infection Control 2021, 10(2): $\bigcirc 46$

Introduction: The 2019-2021 SARS-2 Pandemic has created a number of challenges for healthcare systems globally. In March 2020, the UK NHS looked about to be overwhelmed with the number of patients requiring hospitalisation and intensive care management. The decision was made to create a number of temporary facilities called 'Nightingale Hospitals' in exhibition and conference centres around the UK. The London facility was designed as a 4000 bedded Intensive Care Unit (ICU). This paper describes the challenges in designing, building and running an ICU in an exhibition centre.

Objectives: To describe the design process for a hospital in an exhibition centre. To discuss the challenges experienced during the building process. To describe the challenges faced with staffing such a hospital. To discuss areas in which risk assessments had to be made and practices modified to mitigate suboptimal conditions.

Methods: NHS Designers and Military planners worked in conjunction with the Infection Prevention and Control Team (IPCT) to work with what was available. The whole of the clinical area was deemed to be an Aerosol-generating Procedure (AGP) zone. No oxygen supplies or hot water, minimal access to cold water, almost no drainage and a lack of physical spaces in which to carry out many of the necessary procedures were amongst the challenges faced and were overcome either by design or by changes to optimal practices through mitigation measures. The IPCT had key role to play in ensuring staff and patient safety, including training, stock management of Personal Protective Equipment (PPE) and donning and doffing procedures.

Results: The London Nightingale Hospital became a fully functioning ICU within 10 days of the build commencing and the first patients were admitted within a few days. Thankfully it was only sparingly used as the national lockdown had an effect. In all, 72 patients were admitted with a survival rate of $63 \%$, which was comparable with other established ICUs in London. Transmission rates of COVID-19 in staff were very low in those working clinically. The hospital was closed in June 2020 but reopened in January 2021 in a different guise (rehabilitation) with a smaller number of beds (300) but better facilities as a result of the learning from the first iteration. Conclusion: A temporary hospital built in an exhibition centre successfully managed a number of ICU patients. Lessons learned will shape future planning should this type of facility be required again.

\section{Disclosure of interest}

None declared.

\section{7}

Hand Sanitizer Safety Risks Associated with Poor Formulation and Packaging Approaches: Lessons Learned and Improvements Needed Post-Pandemic

J. Arbogast ${ }^{1, *}$, K. Kucera $^{2}$, D. Light ${ }^{2}$, D. Yeomans ${ }^{1}$, C. Manuel $^{1}$

${ }^{1}$ GOJO, Akron, ${ }^{2}$ Valisure LLC, New Haven, CT, United States

Antimicrobial Resistance \& Infection Control 2021, 10(2): O47

Introduction: The rapid effectiveness of alcohol-based hand rub(ABHR) on microbial pathogens, including viral variants, inspired unprecedented demand for ABHR during the COVID-19 pandemic. A wide variety of new $A B H R$ products that were rapidly produced and released to the public appear to have introduced new and unacceptable human safety risks.

Objectives: While the long-term public health impact of exposure to adulterated $A B H R$ will require further study, the immediate need to assess the safety and efficacy of ABHR motivated this study.

Methods: Sampling of ABHR provided to the public in community settings (e.g., by a medical center) occurred across a wide variety of global community settings. When possible, ABHR samples of 30-50 ml were analyzed for: alcohol \%, visible impurities and chemical impurities (i.e., acetal, acetaldehyde, benzene, and methanol). Validated analytical methods (good laboratory practices (GLP) or International Organization for Standardization (ISO) standards) were used for quantitative measurements. Photographs were taken for visual assessment of packaging design + labeling risks.

Results: Alcohol concentrations less than a typical minimum antimicrobial efficacy level of $60 \%$ were measured in $\geq 7 \%$ of the ABHR samples. Unknown visible impurities were observed in $\geq 11 \%$ of samples. Levels of regulated impurities (acetal, acetaldehyde, benzene, and methanol) in excess of US FDA Guidance were observed in $\geq 43 \%$ of samples. Photographs demonstrate numerous risks associated with package design and open refillable ("bulk") dispensers, including no lot code nor expiry, and lack of traceable labeling.

Conclusion: During the COVID-19 Emergency, demand for ABHR prompted new supply sources, followed by inscrutable donations of surplus products. Though unintended, allowing tainted and poor quality ABHR into communities places clinicians and the public at unnecessary risk. Guidance to consumers, businesses, and communities including medical centers, nursing homes, schools, offices, and retail settings, on the proper evaluation, use, and handling of ABHR, and safe disposal of adulterated or suspect product is needed. Regulatory guidance and enforcement addressing dangerous practices evident in this study including refilling of pump bottles/dispensers with a variety of ABHRs is needed immediately to reduce future exposure to unsafe, ineffective, mislabeled or unlabeled $A B H R$ products.

\section{Disclosure of interest}

J. Arbogast Employee of: GOJO Industries (hygiene products manufacturer), K. Kucera Employee of: Valisure LLC, D. Light Employee of: Valisure LLC, D. Yeomans Employee of: GOJO Industries (hygiene products manufacturer), C. Manuel Employee of: GOJO Industries (hygiene products manufacturer).

048

Hospital-Acquired Respiratory Viral Infections While Applying Droplet Precautions On-site (Drops) - Prospective Observational Study During the 2019/20 Influenza Season, Bern, Switzerland M. A. Birrer ${ }^{1, *}$, K. Draps ${ }^{1}$, F. Hobi ', M. Laguardia', A. Andrew' ${ }^{1}$, E. Hofmann?', M. Blatter ${ }^{2}$, M. Luginbühl ${ }^{3}$, M. Perrig ${ }^{4}$, D. Aujesky ${ }^{5}$, J. Marschall' ${ }^{1}$ R. Sommerstein ${ }^{1}$

11. Department of Infectious Diseases, Inselspital, Bern University Hospital, University of Bern, Switzerland, ${ }^{2}$ Department of General Internal Medicine, Tiefenauspital, Bern, Switzerland, ${ }^{3}$ Department of Anesthesiology, Tiefenauspital, Bern, Switzerland, ${ }^{4}$ Department of General Internal Medicine, Inselspital, Bern University Hospital, University of Bern, Switzerland, ${ }^{5} 4$. Department of General Internal Medicine, Inselspital, Bern University HospitalUniversity of Bern, Switzerland, Bern, Switzerland

Antimicrobial Resistance \& Infection Control 2021, 10(2): O48

Introduction: The guideline-driven and widely implemented single room isolation strategy for respiratory viral infections (RVI) such as influenza or respiratory syncytial virus (RSV) can lead to a shortage of hospital beds. Alternative strategies to prevent hospital-acquired respiratory viral infections (HARVI) are needed.

Objectives: Based on promising results of a pilot study using droplet precautions on-site (DroPS) during the influenza season 2018/19, this strategy was implemented in multiple hospital wards. We assessed the "real-life" safety of DroPS by measuring the rate of HARVI after its implementation.

Methods: Prospective observational study during the influenza season $2019 / 20$ at a tertiary and secondary referral hospital. The study was prematurely stopped at the beginning of the COVID-19 pandemic (17th March 2020). DroPS was used for patients hospitalised with suspected or proven RVI. Hospitalised patients with no respiratory isolation in the two days following admission were considered "at risk" for the acquisition of HARVI. They were screened daily for the onset of new respiratory symptoms. Once an "at risk" patient developed symptoms, an influenza/RSV molecular rapid test was performed. The two 
main outcomes were the rate of clinical and laboratory-confirmed HARVI (influenza or RSV).

Results: We included 1'996 hospitalisations with a total of 8'955 "at risk" hospital days for HARVI. Median age was 71 years [IQR 56-81]. HARVI was clinically diagnosed in $11 / 1^{\prime} 996(0.6 \%)$ hospitalisations. All patients with clinical diagnosis were microbiologically screened and three confirmed $(0.15 \% ; 2 \times$ RSV, $1 \times$ influenza B).

Conclusion: Droplet precautions on site (DroPS) may represent a safe, simple and resource-saving alternative to the traditional pathogenbased single room strategy for RVI in non-pandemic circumstances.

\section{Disclosure of interest}

None declared.

\section{9}

Attitudes, Practices and Sources of Information Towards COVID-19 Vaccination in a Sample of Italian Healthcare Workers

F. Papini', S. Mazzilli², D. Paganini', L. Rago ', G. Arzilli', A. Pan ${ }^{3}$, A. Goglio ${ }^{4}$, B. Tuvo ${ }^{1,}$, , G. P. Privitera ${ }^{1}$, B. Casini ${ }^{1}$

${ }^{1}$ Department of Translational Research and New Technologies in Medicine and Surgery, University of Pisa, ${ }^{2}$ Scuola Normale Superiore, Pisa, ${ }^{3}$ Infectious diseases, ASST Cremona, Cremona, ${ }^{4}$ Scientific Board of the Italian Multidisciplinary Society for the Prevention of Infections in Healthcare Organizations, SIMPIOS, Bergamo, Italy

Antimicrobial Resistance \& Infection Control 2021, 10(2): O49

Introduction: Vaccination of Healthcare workers (HCWs) is a crucial element of the strategy against COVID-19 to conduct an effective immunization campaign among general population.

Objectives: A cross-sectional survey was conducted to assess attitudes, sources of information and practices among HCWs in relation to COVID-19 vaccination.

Methods: From February 19th to April 23rd 2021 an anonymous voluntary questionnaire was sent to the mailing list of SIMPIOS and to the main National Health Service structures. Data collected through the Survey-Monkey platform was subjected to univariate and multivariate analysis to identify factors significantly and independently associated with the variables of interest.

Results: $2137 \mathrm{HCWs}$ answered. Hesitancy is greater in females, in those with lower concern about COVID-19, in nurses, auxiliary nurses (AN) and healthcare assistants. The most hesitant professionals are more likely not to recommend vaccination to either their patients or their relatives, while concern about COVID-19 increases the practice of recommending vaccination to family members, but not to patients. HCWs are mostly in favor of mandatory vaccination (61.22\%). Female sex, a lower education level, greater hesitancy and refusal to adhere to flu vaccination campaigns are factors influencing the aversion to mandatory vaccination. Institutional source of information (eg. World Health Organization) are the most used by all categories. Scientific literature is more used by professionals working in the northern regions of Italy and by those dealing with infection control and hospital hygiene, infectious diseases, emergencies and critical area. HCWs working in south-central regions, nurses, AN, healthcare technicians, administrators and HCWs with a lower education level are more likely to choose internet, television, newspapers, family and friends' advice as sources of information.

Conclusion: Communication in support of COVID-19 immunization campaigns should consider the differences between the various HCWs professional categories, to reach all professionals in a homogeneous way, involving even the most hesitant ones.

\section{Disclosure of interest}

None declared.

Poster session: Antimicrobial resistance-Surveillance

\section{P008b}

Impact of Daily Infection Control Rounds in Intensive Care Unit (ICU) of a Newly Set Up Tertiary Oncology Centre in North India V. Bajpai ${ }^{1,}$, S. Chakravorty ${ }^{1,2}$, D. Pandey ${ }^{3}$, J. Kirtania ${ }^{4}$

${ }^{1}$ Microbiology\& Infection Control, ${ }^{2}$ Surgical Oncology, ${ }^{3}$ Tata memorial
Center, Varanasi, Varanasi, India, ${ }^{4}$ Critical care Unit, Tata memorial Center, Varanasi, Varanasi, India

Antimicrobial Resistance \& Infection Control 2021, 10(2): P008b

Introduction: Regular rounds by Infection control team is important to convince the staff that the principles of infection control in the ICU are based on simple concepts of surveillance and these are the basic measures that are easy to implement at the bedside to prevent hospital acquired infections.

Objectives: This study will evaluate the impact of daily infection control rounds in Intensive Care Unit (ICU) of a newly set up tertiary oncology centre.

Methods: This is a prospective observational study conducted in the Surgical-ICU of MPMMCC Varanasi, India. This is 350 bedded tertiaryoncology-centres,fully commissioned on January-2021.Checklist for daily-ICU-rounds was prepared by using CDC guidelines and daily round was taken by Infection control nurse. Following strategies were considered in the checklist; biomedical waste, Personal- protectiveequipment measure, Environmental-cleaning, hand-hygiene-audit and Device- maintenance-checklist. All variables were analysed to check the compliance or non-compliance of each strategy in the ICU.

Results: $30 \%$ observations of BMW were noncompliant (NC). Most common was mixing of BMW (12\%) followed by improper disposal $(10 \%)$ and overfilling of carts (8\%). Sharp disposal was improper in one-third observations mainly due to wrong disposal (60\%) followed by uncovered-sharp (30\%). PPE measures were followed in $99.3 \%$ times. NC was mainly seen in mainly doctors (6.5\%). Adherence to environmental-cleaning seen in only $47 \%$ observations which was mainly due to NC activity seen in preparation of fresh-hypochloritesolution. Adherence to device maintenancedone in $67 \%$ patients. There is decrease-rate of CLABSI and CAUTI in ICU (CLBASI Rate:9.01 to 0.00 , CAUTI rate (4.78-0.)/1000 patient days. Maximum hand hygiene compliance was observed among nursing officer $(60.4 \%)$ followed by Technician $(41 \%)$ and Doctors (26\%). Maximum attempt of handhygiene were done before and after touching patients seen in nurses (46\%, 50\%),followed by technician (42\%, 33\%) and doctors (33\%, 33\%). Conclusion: BMW,environmental cleaning, hand hygiene activity are essential pillar of strengthening of ICP in ICU. This study strongly confirm that prevention of HAI in ICU can be effective by daily round by Infection control team.

Disclosure of interest

None declared.

\section{P008e}

Fungal Carriage of the External Auditory Canal of Patients in Ear, Nose and Throat Clinic at the University Hospital of Angré, Côte d'Ivoire

M. A. E. A. N'guiachi ${ }^{1, *}$, E. ANGORA ${ }^{2}$, M. QUATTARA ${ }^{3}$, L. KOFFI ${ }^{4}$, A. AHUI

AHUI ${ }^{5}$, V. DJOHAN ${ }^{6}, M^{\prime}$. C. NIAMKE ${ }^{7}$, A. KACOU N'DOUBA ${ }^{8}$

${ }^{1}$ University Hospital of Angré, ${ }^{2}$ parasitology et Mycology

Departement,UFR SPB of Felix Houphouet- Boigny University, ervice of Medical Biology,University Hospital of Angré, ${ }^{3}$ UFR SPB of Felix

Houphouet-Boigny University, ${ }^{4}$ UFR Médical Science Abidjan, ${ }^{5}$ Service of Medical Biology,University Hospital of Angré, ${ }^{6}$ Parasitology et Mycology Departement,UFR SPB of Felix Houphouet Boigny University, UFR SPB of Felix Houphouet Boigny University, ${ }^{7}$ Serviceof Ear Nose and Throat clinic University hospital of Angré, ${ }^{8}$ microbiology department of biology and basic science UFR medical sciences of Felix Houphouet Boigny University, Service of Medical Biology, University Hospital of Angré, ABIDJAN, Côte d'Ivoire

Antimicrobial Resistance \& Infection Control 2021, 10(2): P008e

Introduction: Otomycosis is an infection of the external auditory canal.The fungal agents that colonize this canal can lead to complications. The knowledge of auricular fungal carriage could be a good indicator for mycological surveillance.

Objectives: The objective of this study was to determine the frequency of fungal strains colonizing the external auditory canal in patients at the University Hospital of Angré. 
Methods: This cross-sectional study was carried out among patients who came to Ear, Nose and Throat clinic at the University Hospital of Angré from February to June 2021. For each patient, ear sample was taken using sterile swabs and transferred to the Parasitology-Mycology Unit for mycological analysis.Direct examination and culture on Sabouraud-Chloramphenicol medium were performed for each sample. The identification and antifungal sensitivity of strains were done using the VITEK 2 Compact $^{\circledR}$.

Results: Of 140 samples, 19(13.8\%) yielded positive cultures 13.8\%.Candida parapsilosis was the most isolated yeast species $(57.0 \%)$ while Aspergillus flavus (21.0\%) was the common mold identified.C. albicans and C. parapsilosis were sensitive to antifungals tested while $C$. famata and C. tropicalis were resistant to caspofungin and micafungin. Fungal species identified was correlated with bathing $(p=0.025)$ and ear pruritus $(p=0.023)$.

Conclusion: Fungal colonization of external auditory canal revealed various species with a predominance of $C$. Parapsilosis. An emergence of yeast strains resistant to antifungal was observed.Mycological surveillance of fungi isolated from ear should be managed in further studies using phenotypic and genotypic profiles.

Keywords:External auditory canal-Colonization - Fungi - Antifungal Disclosure of interest

None declared.

\section{P008d}

Not All Multi-drug Resistant Organism (MDRO)S are AlikeLessons from Candida Auris in Singapore

S. Sengupta ${ }^{1, *}$, R. Sridhar ${ }^{1}$, N. Tee ${ }^{2}$, P. A. Tambyah ${ }^{1}$, D. Fisher ${ }^{1}$, J. Somani ${ }^{1}$

${ }^{1}$ General Medicine Infectious Disease, ${ }^{2}$ Microbiology, National University Hospital, Singapore, Singapore

Antimicrobial Resistance \& Infection Control 2021, 10(2): P008d

Introduction: Singapore has seen the emergence of Candida auris as an emerging multi-resistant fungal infection while other MDROs are endemic here.

Objectives: A clinical audit was conducted to measure the efforts to prevent and control transmission of $\mathrm{C}$ auris.

Methods: A retrospective audit of all patients in the hospital epidemiology database diagnosed with C auris from April 2019 to June 2021 was conducted. Prior to Sept 2020, screening for C.auris was done using swabs of nares, axilla and groin of all patients coming from overseas/with recent overseas hospital stay, and contact tracing of newly diagnosed $\mathrm{C}$ auris patients. Screening swabs were cultured by standard microbiological methods (CLSI). After Sept 2020, all patients infected/colonized with carbapenem resistant Enterobacteriaceae (CRE) were also screened for C. auris. Methicillin resistant Staphylococcus aureus (MRSA) screening is routinely done at admission, ward transfer and before certain surgeries while CRE screening is done using a questionnaire. Data was analysed using Microsoft Excel.

Results: Ten patients were diagnosed with $C$ auris infection/colonization from April 2019 to June 2021. Two of 74 (2.7\%) were found from screening of CRE patients and none from overseas screening. An additional case screened positive from contact tracing. Nine of the C. auris isolates were Clade I, the last one was not from any known clade. Nine $C$ auris patients were in single rooms at detection and only one from open ward. In contrast, over the last month, 28 nosocomial MRSA acquisitions identified were all from open wards and none from single rooms.

Conclusion: We found that $2.7 \%$ of patients infected/colonized with CRE were also colonized with Candida auris. Unlike new MRSA colonization, new $C$. auris cases occurred commonly in single rooms rather than open wards $(p<0.0001)$. While $C$. auris has some similarities with other MDROs which can guide screening strategies, there are clearly differences that should be explored in large multi-center prospective studies.

\section{Disclosure of interest}

None declared.
P008c

Research of Antimicrobial Activity of Selected QAC Antiseptics Available for the Prevention and Treatment of the Wound Infection

T. V. Denysko 1,* , N. Bahniuk' , R. Chornopyshchuk², A. Gruzevskiy ${ }^{3}$, H.

Nazarchuk ${ }^{4}$

${ }^{1}$ Microbiology Department, ${ }^{2}$ General Surgery Department, National Pirogov Memorial Medical University, Vinnytsia, ${ }^{3}$ Microbiology Department, Odessa National Medical University, Odessa, ${ }^{4}$ Department of Ophthalmology, National Pirogov Memorial Medical University, Vinnytsia, Ukraine Antimicrobial Resistance \& Infection Control 2021, 10(2): P008C

Introduction: With the global growth of antibiotic resistance, the use of effective antiseptics is becoming the most urgent for successful topical antimicrobial management.

Objectives: The aim was to study the activity of widely used QAC antiseptics against the dominant pathogens of infectious complications of burns and wounds in surgery.

Methods: The solutions of commercially available antiseptics were tested: chlorhexidine digluconate (CHX, 0.05\%), decamethoxin (DCM, 0,02\%), octenidine dihydrochloride $(\mathrm{OCT}, 0.1 \%)$, polyhexanide (polyhexamethylene biguanide, PHMB, $0.1 \%$ ). The susceptibility of reference and clinical strains of S.aureus $(n=40)$, E.coli $(n=38)$, K.pneumonia $(n=26)$, P.aeruginosa $(n=43)$, A.baumannii $(n=42)$ was studied to antiseptics, using the standard broth macro-dilution test. The minimal inhibitory (MIC) and bactericidal (MBC) concentrations were registered and compared.

Results: All microorganisms were found as susceptible to antiseptics. DCM had high biocide efficacy against S.aureus $(\mathrm{MBC}=4.78 \pm 1.31$ $\mu \mathrm{g} / \mathrm{ml})$, E.coli $\quad(\mathrm{MBC}=10.88 \pm 0.79 \mu \mathrm{g} / \mathrm{ml}), \quad$ K.pneumonia $(\mathrm{MBC}=21.15 \pm 1.15 \mu \mathrm{g} / \mathrm{ml})$, A.baumannii $(\mathrm{MBC}=38.32 \pm 6.34 \mu \mathrm{g} /$ $\mathrm{ml}$ ), but low efficacy - against P.aeruginosa (MBC $=106.98 \pm 9.28$ $\mu \mathrm{g} / \mathrm{ml}$ ). CHX, OCT had a similar trend. CHX demonstrated more than twice lower activity against S.aureus, E.coli, K.pneumonia, A.baumannii than DCM $(p<0.001)$. P.aeruginosa were found to be less susceptible to DCM, CHX, OCT $(p<0.001)$. The MBC values of OCT for A.baumannii strains were $28.23 \pm 3.73 \mu \mathrm{g} / \mathrm{ml}$. PHMB demonstrated twice higher bactericidal properties against P.aeruginosa $(52.08 \pm 6.56$ $\mu \mathrm{g} / \mathrm{ml}$ ) comparably to other antiseptics $(p<0.001)$, was alike effective for E.coli, K.pneumonia, but showed twice lower activity against A.baumannii. Poor anti-staphylococcal activity was found in PHMB $(\mathrm{MBC}=179.69 \pm 59.57 \mu \mathrm{g} / \mathrm{ml})$.

Conclusion: Reference and clinical strains of pathogens of the first and second priority categories by the WHO demonstrate high susceptibility to QAC antiseptics, with predominance of DCM activity against S.aureus, E.coli, K.pneumonia, A.baumannii and PHMB against P.aeruginosa $(\mathrm{p}<0.001)$.

Disclosure of interest

None declared.

Poster session: Surgical site infections

\section{P079c}

Patients Perception and Experience on Universal Decolonization with Polihexanide

T. Kramer ${ }^{1, *}$, B. Metzner ${ }^{1,2}$, M. Trojanowski ${ }^{2}$, M. Behnke ${ }^{1}$, C. Schroeder ${ }^{1}$, P. Gastmeier $^{1}$

${ }^{1}$ Charité Universitätsmedizin Berlin, Institute for Hygiene and Environmental Medicine, ${ }^{2}$ Orthopedic Surgery and Traumatology, Evangelisches Waldkrankenhaus Spandau, Berlin, Germany

Antimicrobial Resistance \& Infection Control 2021, 10(2): P079C

Introduction: Surgical site infections (SSI) are one of the most severe complications in joint arthroplasty. Decolonization measures prior to elective orthopedic surgeries have been shown to reduce the risk of SSI especially in patients colonized Staphylococcus aureus. Implementation is now recommended by international and national guidelines. However knowledge on compliance, perceptions and acceptance among patients undergoing surgery is scarce. 
Objectives: The objective of our study was to investigate the perception and experiences of patients on preoperative managament in patients participating in a trial implementing universal decolonization with polihexanide.

Methods: Patients scheduled for hip or knee joint arthroplasty in 5 participating orthopedic centers were included between January 2017 and December 2018. Patients that received decolonization sets containing polihexanide and consented to participate in the study were asked to answer a questionnaire consisting of 14 closed items with a 5 point Likert scale. Patients received the questionnaire on the day of their preoperative visit and were asked to return it in an envelope to nursing staff upon admission.

Results: Of 1866 patients that consented to participate in the study $1381(74.0 \%)$ returned the questionnaire. Of those 1333 patients (96.5\%) answered that they were at least in part compliant with the protocol and used the set. 1221 of the patient felt that they were sufficiently informed about application and potential adverse effects. 1224 patients used the decolonization set according to manufacturer's recommendations. 239 patients were not able to use the set without help. 251 patients had mild to moderate adverse events. Dryness of skin and flavor of oral solution were most frequently noted. Additional factor on the patients experience were investigated.

Conclusion: Patients felt well informed about potential risk and general complications prior to their surgery. They are willing to perform additional task in preparation. Implementation of universal decolonization created a low number of adverse events.

\section{Disclosure of interest}

T. kramer Grant /Research support from: B.Braun, B. metzner: None declared, M. trojanowski: None declared, M. behnke: None declared, C. schroeder: None declared, P. gastmeier: None declared.

\section{Poster session: COVID-19: Environmental control}

\section{P099b}

Antimicrobial Photodynamic Disinfection (APDT) Destroys SARS-COV-2 Receptor Binding Domains and Spike Protein Independent of Viral Variant

C. Romo ${ }^{1}$, N. Loebel ${ }^{1, *},{ }^{*}$ C. Cross ${ }^{1}$, R. Andersen ${ }^{1}$

'Ondine Research Labs, Bothell, United States

Antimicrobial Resistance \& Infection Control 2021, 10(2): P099b

Introduction: We previously reported on the elimination of the RNA signature of laboratory strains as well as wild-type SARS-CoV-2 using a photodynamic disinfection technique. This report extends the work to destruction of receptor binding domains, spike protein and nucleocapsid protein of major SARS-CoV-2 variants including the dominant Delta variant.

Objectives: The objective of this work was to evaluate effect of a 2-min cycle of aPDT on receptor binding domains, spike protein and nucleocapsid protein of SARS-CoV-2.

Methods: Spike glycoprotein receptor binding domain proteins from SARS-Related Coronavirus 2 included recombinant NR-54004 (United Kingdom Variant), NR-54005 (South African Variant), and NR-52366 (Wuhun-Hu-1 Variant) (BEI Resources, NIAID, ATCC). Recombinant spike proteins included B.1.1.7 Spike Protein, United Kingdom Variant (10748-CV-100), B.1.617 Spike Protein, Indian (Delta) Variant (10861CV-100), B.1.351 Spike Protein, South African Variant (10777-CV-100), P.1 Spike Protein, Brazilian Variant (10795-CV-100) (all from R\&D Systems, Inc.), and the stabilized spike glycoprotein, Wuhun-Hu-1 Variant (NR-52397). The SARS-CoV-2 nucleocapsid protein (NR55344, Avi-Histag, Biotin-Labeled, BEI Resources, NIAID, ATCC) was also tested for susceptibility to damage. aPDT was carried out by exposing each viral component to a photosensitizer formulation containing $320 \mathrm{uM}$ methylene blue in an aqueous adjuvant, immediately followed by $36 \mathrm{~J} / \mathrm{cm}^{2}$ $(120 \mathrm{~s})$ of non-thermal laser light at $664 \mathrm{~nm}$ (Steriwave ${ }^{\circledR}$ aPDT system, Ondine Biomedical Inc., Vancouver, B.C.). Illumination was conducted in a custom thin-film cell replicating the anterior nasal architecture. Control solutions were identically treated but without light exposure. $50 \mathrm{ul}$ samples of treated and control solutions were evaluated by SDSPAGE using SYPRO Ruby Protein Gel Stain.
Results: SDS-PAGE evaluation of all treated RBD, spike and SARSCoV-2 nucleocapsid protein samples showed no detectable protein remaining after 2 min exposure to photodynamic disinfection treatment. All no-light control samples remained unaffected.

Conclusion: A 2-min photodynamic disinfection procedure was demonstrated to destroy RBD's, spike protein and nucleocapsid protein of major SARS-CoV-2 viral variants in circulation, expanding on previous work evaluating RNA damage in this virus. This outcome supports the use of aPDT as a potential SARS-CoV-2 suppression technique.

Disclosure of interest

C. Romo Employee of: Ondine Research Labs, N. Loebel Employee of: Ondine Research Labs, C. Cross Employee of: Ondine Research Labs, R. Andersen Employee of: Ondine Research Labs.

\section{Poster session: COVID-19: Vaccination}

\section{P116b}

COVID 19 Vaccine and Cancer: A Prospective Study of 186 Cases

W. Ben Kridis ${ }^{1, *}$, A. Feki ${ }^{1}$, S. Khmiri ${ }^{1}$, N. Toumi ${ }^{1}$, A. Khanfir ${ }^{2}$

${ }^{1}$ Department of oncology sfax tunisia, ${ }^{2} \mathrm{Habib}$ Bourguiba Hospital, Sfax,

Tunisia

Antimicrobial Resistance \& Infection Control 2021, 10(2): P116b

Introduction: Vaccination against SARS-CoV-2 is currently recommended. Cancer patients are considered a priority. In Tunisia, these patients were included on the vaccination platform as priorities. The only contraindication to this vaccine is neutropenia.

Objectives: The objective of this work was to study the acceptability and adherence of the COVID 19 vaccine in cancer patients.

Methods: This is a prospective study including cancer patients undergoing chemotherapy. The study was carried out at the medical department of oncology at Habib Bourguiba hospital in Sfax between March 2021 and June 2021.

Results: 186 patients were included including 38 men and 148 women. The average age was 52 years old [24-72 years old]. The site of the tumor was mammary in $61.3 \%$ of cases, recto-colic in $11.3 \%$, and at the cavum in $3.8 \%$. The tumor was localized in $52.7 \%$ and metastatic in $47.3 \%$ of cases. The chemotherapy was neoadjuvant in $9.1 \%$ of cases, adjuvant in $44.1 \%$ of cases and palliative in $46.2 \%$. Administration of targeted therapies was observed in $25.3 \%$ of patients. Ten patients (5.3\%) have a COVID infection during their treatment. Ninety-nine patients $(53.2 \%)$ accepted the vaccine. Sixty-nine patients (37.1\%) refused and eighteen patients $(9.7 \%)$ were hesitant. Thirty patients $(16.1 \%)$ were enrolled at the vaccine registration site. Twenty patients $(10.7 \%)$ had the SARS-CoV-2 vaccine. The tolerance of the vaccination was good.

Conclusion: It is important to vaccinate all cancer patients under chemotherapy given the severity of the COVID-19 infection and this after checking the blood count. In our series, $53.2 \%$ of patients agreed to have the COVID19 vaccine. This rate was higher than that described in the literature which is $37.4 \%$. This can be explained by the awareness of patients through the vaccination brochures.

Disclosure of interest

None declared.

Poster session: COVID-19 among healthcare workers

P136b

Investigation and Control of Covid-19 Infections Among Healthcare Workers (HCWS) in a Covid-19 Department - Lessons from an Outbreak

K. Labay ${ }^{1, *}$, I. Aharon ${ }^{1}$, D. Glikman ${ }^{1,2}$, H. Mizrahi ${ }^{2,3}$, H. Zayyad ${ }^{1,2}$

${ }^{1}$ Infectious diseases unit, Baruch Padeh Medical Center, Tiberias, ${ }^{2}$ Faculty of Medicine, Bar Ilan University, Sefad, ${ }^{3}$ Baruch Padeh Medical Center,

Tiberias, Israel

Antimicrobial Resistance \& Infection Control 2021, 10(2): P136b 
Introduction: At our 350-bed hospital in Northern Israel, the COVID19 department is based on site of an internal medicine ward. At the second wave of COVID-19 in Israel, hospitalized patients were mostly severe-to-critical, needing high flow supplemental Oxygen (HFNC) or mechanical ventilation (MV). During September 2020, 13.3\% of HCWs in the ward were infected with COVID-19. This was out of proportion compared to infections among HCWs in other departments and in the community and warrented action.

Objectives: To investigate and control the outbreak among HCWs.

Methods: 1. Investigating the source of infection: the infection prevention and control (IPC) team categorized the presumable source of infection as: (A) out of hospital. 2. Direct observations of department staff on doffing and donning PPE; time spent in the COVID area. 3. Screening HCWs for SARS-COV2, all tested HCWs filled a questionnaire about their workflow in the ward. 4. Inspection of the ward building structure with emphasis on air flow and air exchanges.

Results: Twelve HCWs $(12 / 90,13.3 \%)$ were infected with COVID-19 during September 2020, all were symptomatic, none hospitalized. Source of infection was categorized as (C) for $75 \%$ of infected HCWs. No major issues with the doffing process were found. $98 \%$ of the HCWs performed a leak test upon use of $\mathrm{N} 95$ masks, 63\% reported fitting problems. $75 \%$ of the infected staff spent more than $2 \mathrm{~h}$ in the COVID19 area at a time. Four different kinds of N95 masks were used. Three of these masks were found not compatible for use and were disqualified by the IPC staff. The ward constituted of a clean area and a COVID area: after donning PPE in the clean area, COVID area is entered using an anteroom, HCWs use a different anteroom for doffing then back to the clean area. Only 2 air exchanges per hour in each room, with no negative pressure rooms. Corrective measures lead to control of the outbreak and included 2 main interventions: (1) using only N95 masks which were tested using standardized methods, and approved by the IPC team (2) improving ventilation system: increasing air exchanges and one-way-air-flow from clean to COVID area.

Conclusion: Appropriate PPE and improvement of the ventilation and airflow in COVID-19 department lead to outbreak control. These actions improved staff safety and confidence among HCWs during patient care.

\section{Disclosure of interest}

None declared.

\section{Poster session: SARS-CoV2: PPE}

\section{P157b}

Probable Cases Evacuation-Body Top Jacket with Portable Hepa Filter

W. K. Poon ${ }^{1,2,}$, C. H. Cheng ${ }^{3}$, H. C. Leung ${ }^{3}$, W. M. Kai ${ }^{3}$ on behalf of KAR ${ }^{1} \mathrm{AEMAU}$, The Chinese University of Hong Kong, ${ }^{2}$ Health \& Science Disciplines, IVE/ Kwai Chung, ${ }^{3}$ Central Nursing Division, Hospital Authority, Hong Kong SAR, China

Antimicrobial Resistance \& Infection Control 2021, 10(2): P157b

Introduction: In view of the latest covid-19 pandemic condition worldwide, it affects greatly on paramedical colleagues daily practice especially on the suspected or not yet confirmed case transfer or evacuation. Unlike those server deadly infectious diseases case which goanna to have strict isolation devices, and most patients condition are ambulatory or transfer for isolation purposes only.

Objectives: To develop a head to abdomen enclosed well covered jacket for transfer purposes in order to reduce the chance of droplets and aerosols spread whilst patient transfer to strict isolation areas. Thus, patient should feel comfortable and acceptable during the process.

Methods: Soft plastic core shell on back and with hard transparent front was built; and loosely tight seal on all sides via elastic wrapper. Besides, airs will be sucked through HEPA filter to eliminate any infectious droplets or aerosols leak out to open air and infect those paramedical colleagues who simply escort the case to major Hospitals or strict isolation facility.
Results: Laboratory test conducted for its antimicrobial activities against Pseudomonas Aeruginosa (ATCC No. 9027), Enterobacteriaceae (Escherichia coli ATCC No. 8739); Staphylococcus aureus (ATCC No. 6538P); ISO 22196: 2011 Measurement of antibacterial activity on plastics and other non-porous surfaces methodology adopted and result indicated with its compliance of the antimicrobial activities. Trial run successfully with the simulated patient transfer and the safety standards as HEPA filter efficiency minimal standard well-kept in order to maintain the air seal internal environment via HEPA suctioning. In order to maintain the front shape for easy observation and comfort, it remodeled into hard shell. Jacket shell is used environmental friendly materials for disposable, combustible as of non-polyvinylchloride material.

\section{Figure / Image:}

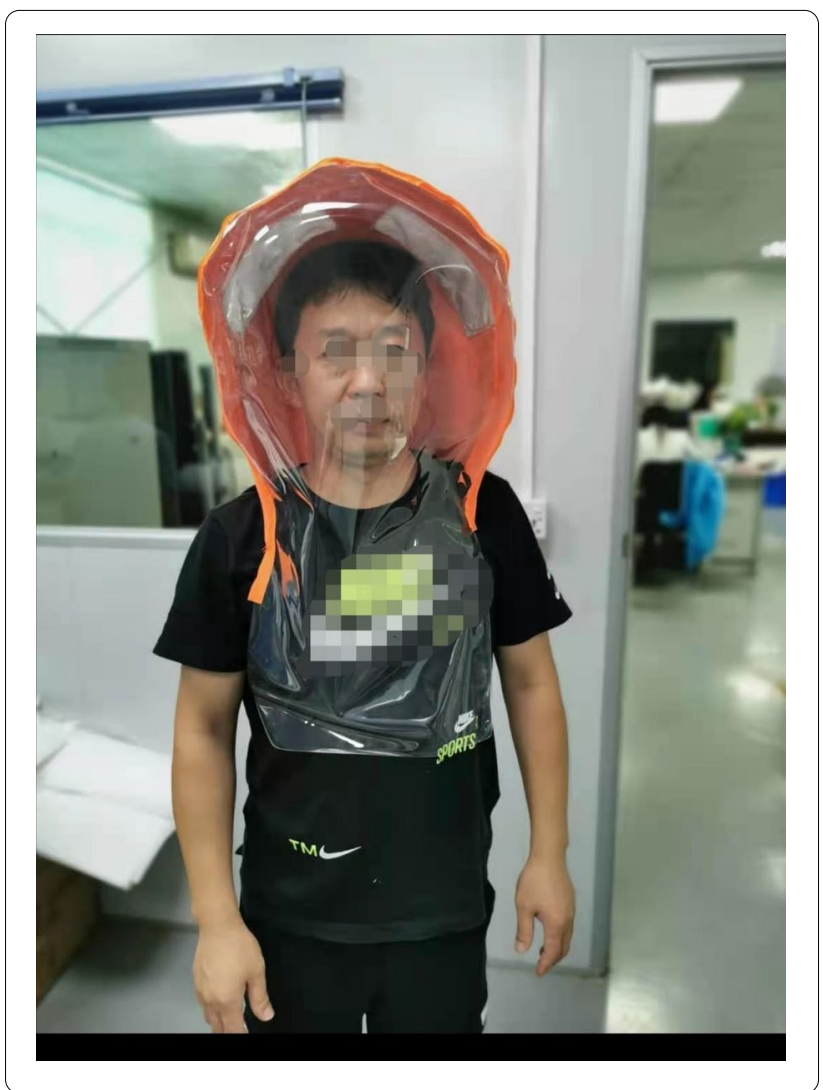

Conclusion: Transfer Jacket enhances safety for health care providers as well as transportation vehicles immediate environment plus peoples nearby with an extra protection. Besides comfort of the evacuee can be enhanced and safety ranges and aspects consideration are also being well taken care of.

Disclosure of interest

None declared.

Poster session: Environmental control: Medical devices

P190b

GISIO-SIMPIOS Multicenter Study on Post-reprocessing Microbiological Surveillance of Flexible Endoscopes used in Digestive Endoscopy: Preliminary Results B. Casini ${ }^{1,{ }^{*}}$, M. Sartini ${ }^{2}$, B. Tuvo ${ }^{1}$, A. M. Spagnolo ${ }^{2}$, D. Vay ${ }^{3}$, A. Segata ${ }^{4}$, B. Mentore $^{5}$, S. Vincenti ${ }^{6}$, M. De Giusti ${ }^{7}$, E. Vecchi ${ }^{8}$, L. Baroncelli ${ }^{9}$, M. M. Distefano ${ }^{10}$, P. Laganà ${ }^{11}$, I. Torre ${ }^{12}$, P. Castiglia ${ }^{13}$, M. L. Cristina ${ }^{2}$

${ }^{1}$ Dept. Translational Research, N.T.M.S., University of Pisa, Pisa, ${ }^{2}$ Dept. 
Health Sciences, University of Genoa, Genoa, ${ }^{3}$ Azienda Sanitaria Ospedaliera S.S. Antonio e Biagio e C. Arrigo, Alessandria, ${ }^{4}$ Azienda Provinciale per i Servizi Sanitari di Trento, Trento, ${ }^{5}$ ASL4 Lavagna, Genoa, ${ }^{6}$ Fondazione Policlinico Universitario Agostino Gemelli IRCCS, ${ }^{7}$ Azienda Ospedaliera Universitaria Policlinico Umberto I, Roma ${ }^{8}$ Azienda Ospedaliero-Universitaria di Modena, Modena, ${ }^{9}$ Ospedali Riuniti di Livorno, Livorno, ${ }^{10}$ Azienda Ospedaliera per l'Emergenza Cannizzaro, Catania, ${ }^{11}$ Dept. BIOMORF, University of Messina, Messina, ${ }^{12}$ Dept. of Public Health, University of Naples Federico II, Naples, ${ }^{13}$ Dept of Medical, Surgical and Experimental Sciences, University of Sassari, Sassari, Italy

Antimicrobial Resistance \& Infection Control 2021, 10(2): P190b

Introduction: Several epidemic events caused by multi-resistant microorganisms following endoscopic procedures were reported despite strict adherence to endoscope reprocessing guidelines.

Objectives: A nationwide cross-sectional study was conducted by the members of the scientific societies SITI (GISIO working group) and SIMPIOS to verify the effectiveness of reprocessing procedures of duodenoscopes.

Methods: An ad hoc questionnaire was designed for collecting data about the management and activity of the endoscopy centers and those related to devices and their reprocessing and storage. Microbiological surveillance was conducted according to the "Duodenoscope Surveillance Sampling and Culturing Protocol", CDC 2018. Statistical analysis was carried out by the STATA SE14TM software (StataCorp LP-USA) and relationships between data were examined by the Spearman's rank correlation test.

Results: Fifteen centers uniformly distributed in Italy participated, with a total of 144 post-reprocessing microbiological samplings performed on 55 duodenoscopes, of which $39.6 \%$ were found to be non-compliant, with high-concern (HC) and low-concern (LC) microorganisms detected in $29.2 \%$ and $10.4 \%$ of samples, respectively. The most frequently detected HC microorganisms were Pseudomonas aeruginosa (16\%), followed by Escherichia coli (16\%) and Klebsiella pneumoniae $(14 \%)$. The mean value of microbial load relative to HC microorganisms was $441 \pm 1196$ (CFU/duodenoscope), with minimum and maximum values of 1 and $5400 \mathrm{CFU} /$ duodenoscope. A statistically significant correlation was found between the microbial load of HC microorganisms and the storage time in non-ISO 16442 compliant cabinets before sampling ( $r h o=0.6834, p<0.05)$.

Conclusion: The preliminary results of the multicenter study showed a significant percentage (39.6\%) of non-conformities in the microbiological investigation, incurred mainly due to $\mathrm{HC}$ microorganisms, and, consequently, a potential risk of cross-transmission associated with the use of duodenoscopes.

\section{Disclosure of interest}

None declared.

Poster session: Environmental control: Others

\section{P199b}

Stability of Viral RNA in an Environmental Sampling Kit and its Detection with Molecular Biology Techniques

M. Beffa', S. Paghera', R. Botrugno ${ }^{1}$, C. Sabelli ${ }^{1}$,

${ }^{1}$ Copan Italia SpA, Brescia, Italy

Antimicrobial Resistance \& Infection Control 2021, 10(2): P199b

Introduction: The detection of SARS-CoV-2 on inanimate surfaces lead to an increasing demand for environmental sampling solutions to detect microbiological contaminations. Monitoring of surface contamination was used to monitor potential implication for viral transmission and possible mitigation strategies using effective cleaning and disinfection procedures and precaution.

Objectives: The purpose of this study was to evaluate viral RNA sample stability using Copan SRK ${ }^{\circledR}$, an environmental sampling kit consisting of a pre-moistened swab for sample collection and a reagent able to neutralize antimicrobial agents, for the detection of RNA Influenza A virus with molecular biology techniques.

Methods: For the study, $100 \mu \mathrm{L}$ of Influenza A virus (H3N2) (ATCC ${ }^{\circledR}$ VR-1679 $9^{\mathrm{TM}}$ ) viral suspension, chosed as representative of a ssRNA virus, were inoculated in $1 \mathrm{~mL}$ SRK tubes. To simulate product performances upon shipment, some SRK tubes were previously stressed performing a thermal shock. Additional $100 \mu \mathrm{L}$ were inoculated in Copan UTM as reference. Viral nucleic acids were extracted with the ThermoFisher PrepSEQ ${ }^{\mathrm{TM}}$ Residual DNA kit and the Applied Biosystem Automate express at different timepoints from inoculation. Samples were extracted at T0, after 2 and 7 days of storage at $2-8^{\circ} \mathrm{C}$ and room temperature. Nucleic acids were stored at $-20^{\circ} \mathrm{C}$ until test (all samples were subjected to a freeze/thaw cycle before test). Detection of viral RNA was performed with the RIDA ${ }^{\circledR}$ GENE real time PCR Flu/RSV. Additionally, to assess long term preservation, samples were extracted after 28 days at both $2-8{ }^{\circ} \mathrm{C}$ and RT starting from Copan SRK tubes.

Results: Data obtained (Table 1) showed that Copan SRK is suitable to preserve inoculated samples and viral RNA from Influenza A virus can be detected up to 7 days from collection when stored at $2-8{ }^{\circ} \mathrm{C}$ and RT and up to 28 days at $2-8{ }^{\circ} \mathrm{C}$, with a difference in Ct from T0 lower than $1 \mathrm{Ct}$ (Fig. 1). As reference, samples stored at RT up to 7 days in UTM showed the same variation in Ct values from T0. No significant differences were observed when variation in $\mathrm{Ct}$ values is compared between SRK with and without the stress procedure.

Table 1

\begin{tabular}{|c|c|c|c|c|c|c|c|c|c|c|c|c|}
\hline \multirow[t]{2}{*}{$C_{t}$} & \multicolumn{3}{|l|}{ Day 0} & \multicolumn{3}{|c|}{ After 2 days } & \multicolumn{3}{|c|}{ After 7 days } & \multicolumn{3}{|c|}{ After 28 days } \\
\hline & Mean & SD & & Mean & SD & $\Delta C_{t}$ & Mean & SD & $\Delta \mathrm{C}_{\mathrm{t}}$ & Mean & SD & $\Delta \mathrm{C}_{\mathrm{t}}$ \\
\hline UTM & 27.48 & 0.056 & RT & 28.37 & 0.063 & 0.89 & 28.08 & 0.028 & 0.6 & & & \\
\hline \multirow[t]{3}{*}{ SRK } & 27.52 & 0.150 & RT & 26.76 & 1.367 & -0.76 & 27.15 & 0.257 & -0.37 & 30.13 & 0.240 & 2.61 \\
\hline & & & $2-8^{\circ} \mathrm{C}$ & 27.04 & 0.298 & -0.48 & 27.96 & 0.415 & 0.44 & 28.45 & 0.441 & 0.93 \\
\hline & & & RT & 27.35 & 0.483 & 0.1 & 27.09 & 0.265 & -0.16 & & & \\
\hline $\begin{array}{l}\text { SRK } \\
\text { stressed }\end{array}$ & 27.25 & 0.306 & $2-8^{\circ} \mathrm{C}$ & 26.89 & 0.134 & -0.36 & 27.44 & 0.303 & 0.19 & & & \\
\hline
\end{tabular}

\section{Figure / Image:}

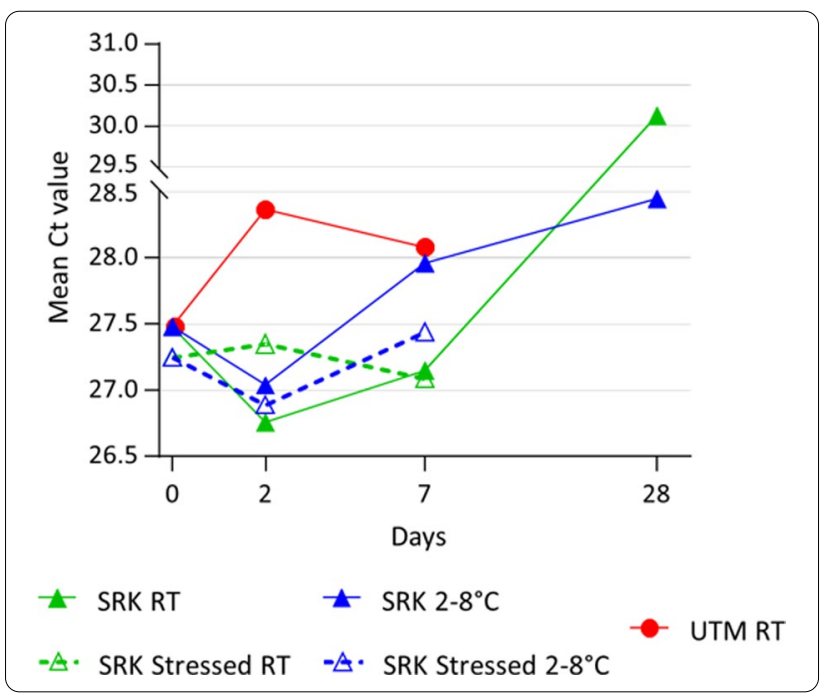

Conclusion: Copan SRK is compatible with the detection of Influenza A viral RNA with molecular biology techniques and samples are stable up to 7 days of storage at $2-8{ }^{\circ} \mathrm{C}$ and RT and up to 28 days at $2-8^{\circ} \mathrm{C}$.

Disclosure of interest

None declared. 


\section{Poster session: Environmental control: Products}

\section{P206b}

Empowering Housekeeping Staff: Evaluation of Routine Inpatient Room Cleaning Using Fluorescent Marker

R. Dayag,

${ }^{1}$ Quality and Patient Safety, Communicable Disease Center, Hamad Medical Corporation, Doha, Qatar

Antimicrobial Resistance \& Infection Control 2021, 10(2): P206b

Introduction: Visual assessment is not enough to ensure quality of the cleaning process. It is necessary to guarantee the level of cleanliness through validation strategy.

Objectives: To develop a method to validate the cleaning process adopted by the housekeeping staff in using fluorescent marker, use of cleaning pathway guide, and gain a better understanding of what they know about the high-touch areas (HTA's).

Methods: HTA's were identified and a checklist was made for data collection. In the first Plan Do Study Act (PDSA) cycle, flourescent solution was applied to the HTA's without informing them. Evaluation of the areas after cleaning resulted in the baseline data. Next, education were done and the use of cleaning pathway guide was introduced. This allows the staff to move through the room without tracking dirt and germs back into the areas they've cleaned and follows the top to bottom and cleanest to dirtiest technique. Simulation was completed by applying the cleaning pathway. Staff's perception and satisfaction for overall cleaning process, knowledge of the HTA's, organisms' transmission-based precaution were identified. The measures taken were percentage of housekeeping compliance to room cleaning pathway and the housekeeping compliance in cleaning HTA's.

Results: Assessment of the current practice shows 20\% (baseline data) of the HTA's are cleaned. PDSA second cycle thrusted the team to develop a training plan to educate the staff on the identification of HTA's and the use of cleaning pathway guide. This gave a post intervention rate of $67 \%$ net improvement $(\mathrm{NI})$ from the baseline data $(87 \%$ compliance rate) and $87 \% \mathrm{NI}$ (100\%compliance rate) at the end of the project. Housekeeping perceptions were also assessed pre and post implementation of the training plan. A NI of $26 \%$ (57\% initial assessment, $87 \%$ post training) was achieved after the initial assessment. Overall, positive housekeeping insight improved their knowledge, skill and awareness thus promoting a safe environment for patient, staff, and visitors alike.

Figure / Image:

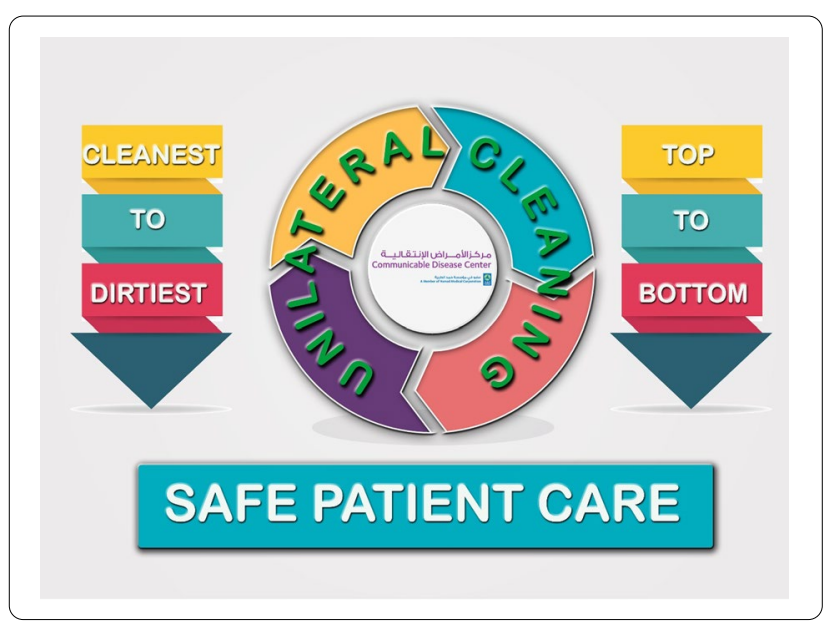

Conclusion: Visually, to say that an area is cleaned is a subjective interpretation. There must be an objective method to validate it. This project was successful in providing a validation system for housekeeping cleaning practices. There was a significant improvement in the housekeeping's perception and practices particularly in knowing the
HTA's and following the cleaning pathway guide to improve cleaning practices.

Disclosure of Interest

None declared.

Poster session: Hand Hygiene: Communication and promotion across the globe

\section{P237b}

Nationwide Online Hand Hygiene Training Program/Competition for Azerbaijani Medical and Health Sciences Students

N. Demiral Y.lmaz 1, , N. Memmedova², O. Karaca' ${ }^{1}$, V. Aghayeva², S. Cavadov $^{2}$, J. Collins ${ }^{3}$, A. P. Rehse Countinho ${ }^{4}$, T. Gulu ${ }^{3}$, H. Harmancl ${ }^{3}$, H. I. Durak $^{3}$

${ }^{1}$ Ege University School of Medicine, Izmir, Turkey, ${ }^{2}$ Azerbaijan Medical School, ${ }^{3}$ World Health Organization, Baku, Azerbaijan, ${ }^{4}$ World Health

Organization, Kopenhagen, Denmark

Antimicrobial Resistance \& Infection Control 2021, 10(2): P237b

Introduction: Healthcare-associated infections (HAl) are the most frequent adverse event in healthcare. Hand Hygiene $(\mathrm{HH})$ is a crucial intervention to prevent the transmission of HAls that should be learned in the early years of education by health care workers. Since COVID 19 pandemic hit education systems worldwide and face-to-face (f2f) training stopped, there is a need for innovative and effective distance educational interventions.

Objectives: This pre-test, post-test intact group experimental design educational intervention study aims to create a unique online $\mathrm{HH}$ training program/competition which motivates students to participate and learn with high satisfaction.

Methods: $\mathrm{HH}$ training program/competition was developed with the involvement of all 8 health sciences faculties and 11 medical colleges' representatives in one f2f[jc1] and two online meetings. Pre/posttests, skills training guides and WHO guidelines-based ppt presentations were developed. Materials were made accessible at the "flipgrid" application. Students registered and attended $2 \mathrm{~h}$ long synchronous pre-test, demonstration sessions through Zoom and Facebook stream followed by a post-test. They then recorded and uploaded their correct handwashing practice. We used Google forms to collect students' feedback ( 0 very bad- 10 very good).

Results: The total target students number was 14.180. 812 and 1412 students completed pre and post-test respectively and 1.900 during the stream. All 212 students who uploaded videos were evaluated, ranked between 0 and 100, receiving written feedback. Post-test scores were high $(x=81.0 \pm 2.3)$. There were significant differences between mean total scores $(t=26.5 ; p<0.000)$ of the pre-post test. The mean score of the evaluated videos was found as $(x=72.0 \pm 1.4) .842$ students provided feedback; general satisfaction was very high $(x=9.57 \pm 1.11)$.

Conclusion: This study suggests that the organization of nationwide online $\mathrm{HH}$ training/competition for health students is feasible and associated with acceptable participation rates and learning levels, with high satisfaction. Technology,internet access and digital literacy levels remain under-researched in this study to explain nationwide $10 \%$ participation.

\section{Disclosure of interest}

None declared.

\section{P237c}

Hand Washing Quality Feedback using a Video Recordings in One of The University Hospital Clinics in Latvia

A. Melbarde-Kelmere ${ }^{1,2,}$, M. Lulla ${ }^{2}$, A. Rutkovskis ${ }^{2}$, A. Elsts ${ }^{3}$, M. Ivanovs ${ }^{3}$, A. Slavinska ${ }^{2}$

${ }^{1}$ Riga East University Hospital, ${ }^{2}$ Riga Stradins University, ${ }^{3}$ Institute of Electronics and Computer Science, Riga, Latvia Antimicrobial Resistance \& Infection Control 2021, 10(2): P237C

Introduction: According the World Health Organization thousands of people impacted every day around the world from infections acquired when they receive health care. Hands are the main source of transmission of infection in health-related care. The guidelines recommend 
manual hygiene following the 6-step method. Manual hygiene monitoring can be carried out using different methods-direct monitoring, video filming, calculating the consumption of hand disinfectant etc.

Objectives: The gold standard is a direct observation of health care worker hand hygiene, but due to pandemic situation, also infection control staff shortage, the new technologies should be implemented in health care for,

Methods: Special data devices were installed in hospital clinics, to record all operations carried out at the sink, including hand-washing movements. For the video recording the IP cameras "POE-100CAMv2 used. Created 8 identical places near sinks (in the procedure rooms, ICU rooms) for obtaining video files near the sink for $24 \mathrm{~h}$ period/7 days a week. A special computer program was used to annotate video files. The developed program allows to encode each video frame with a special code, corresponded to a specific hand-washing movement recommended by the World Health Organization. The video files obtained at the hospital were recorded in principle-the recording is made and stored if a movement of at least $15 \mathrm{~s}$ is detected at the hand-washing site. All data manually reviewed and only hand washing episodes included in the study.

Results: In general, cameras were placed in 8 locations and 14,886 video files were obtained, of which 2120 video was identified as valid for the study (i.e. the video content was hand washing). The rest of the video contained non-manual washing activities such as dishwashing. Medium hand washing time is $13 \mathrm{~s}$. Compliance rate for all hand washing steps is $0.8 \%$. Only 6 video files shows hand washing time more than $40 \mathrm{~s}$.

\section{Figure / Image:}

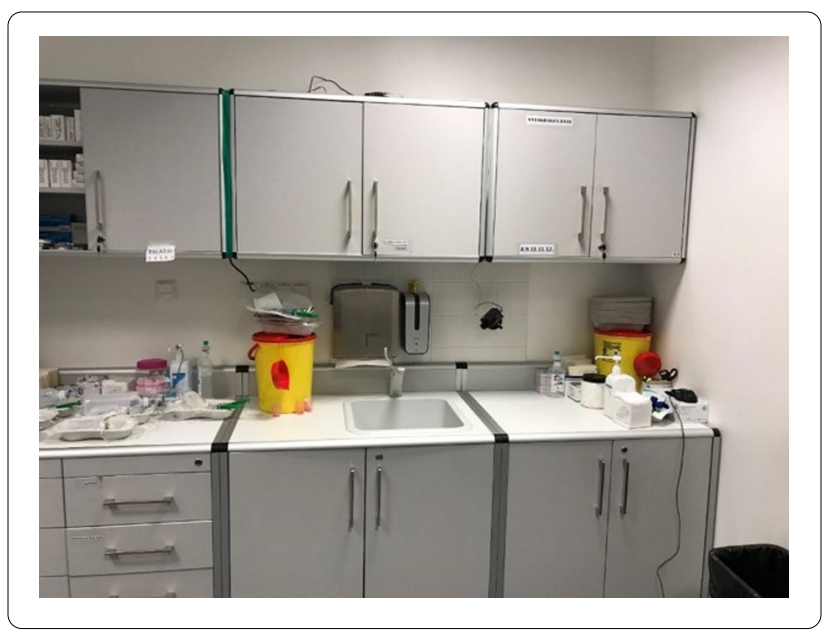

Conclusion: Hand washing compliance is too low, but in the future the Influence of hand washing quality feedback on users behavior using a quality assessment tool based on neural networks/ simulated neural networks will be monitored on next study.

\section{Disclosure of interest}

None declared.

\section{Poster session: COVID-19 outcomes and prediction}

\section{P310b}

The Relationship Between Demographic Factors and Levels of Self-Care Against Coronavirus in Pregnant Women Referred to Maternity Wards

K. Alidousti 1, ${ }^{*}$, F. Moradi ${ }^{2}$, M. GHazanfarpour ${ }^{1}$, A. Hosseininasab ${ }^{3}$, F. SHojaee $^{4}$

${ }^{1}$ Kerman Medical Science University. Midwifery Department, ${ }^{2}$ Kerman Medical Science University.Payambar Aazam Hospital, ${ }^{3}$ Kerman Medical Science University. Pediatric Department, ${ }^{4}$ Kerman Medical Science University. Afzalipour Hospital, Kerman, Iran, Islamic Republic of Antimicrobial Resistance \& Infection Control 2021, 10(2): P310b
Introduction: The adverse effects of coronavirus infection on pregnant women and their infants are not apparent. The best strategies to deal with this disease is avoiding the infection and preventing its transmission.

Objectives: the present study aimed to investigate the relationship between demographic factors and levels of self-care against coronavirus in pregnant women referred to maternity wards of Kerman, Iran.

Methods: The present descriptive study was conducted on 200 pregnant women who referred to maternity wards in Kerman, Iran in 2020 and met the inclusion criteria. The required information was collected using demographic questionnaires and a self-care checklist.

Results: The mean age of the participants was $28.89 \pm 7.07$. Iranian and Afghan citizens comprised $82 \%$ and $18 \%$ of the participants, respectively. The highest level of self-care measures against coronavirus in pregnant women was attributed to the use of face masks (74\%), and the lowest was warning the personnel to wear masks (28\%). There was a statistically significant relationship between the nationality of the participants and warning the personnel to wear face masks $(P=0.02)$, the pregnant mothers' attention to wearing a scarf or cap during labor with the mothers' occupation $(P=0.006)$, having a sick spouse $(P=0.039)$, and having a sick child $(P=0.043)$, and between the patients' husbands' job and the patients' demand for a private room $(P=0.013)$.

Conclusion: The results indicated that most pregnant women in the present study were active in self-care against coronavirus. Using face masks was more widely followed than other self-care measures; moreover, there was a relationship between personal characteristics and self-care levels.

Disclosure of interest

None declared.

\section{Publisher's Note}

Springer Nature remains neutral with regard to jurisdictional claims in published maps and institutional affiliations.
Ready to submit your research? Choose BMC and benefit from:

- fast, convenient online submission

- thorough peer review by experienced researchers in your field

- rapid publication on acceptance

- support for research data, including large and complex data types

- gold Open Access which fosters wider collaboration and increased citations

- maximum visibility for your research: over $100 \mathrm{M}$ website views per year

At $\mathrm{BMC}$, research is always in progress.

Learn more biomedcentral.com/submissions 